\title{
Energy Efficiency Maximization of Full-Duplex and Half-Duplex D2D Communications Underlaying Cellular Networks
}

\author{
Yiliang Chang, ${ }^{1}$ Hongbin Chen, ${ }^{1,2}$ and Feng Zhao ${ }^{1}$ \\ ${ }^{1}$ Key Laboratory of Cognitive Radio and Information Processing, Guilin University of Electronic Technology, Guilin 541004, China \\ ${ }^{2}$ Guangxi Experiment Center of Information Science, Guilin 541004, China \\ Correspondence should be addressed to Hongbin Chen; chbscut@guet.edu.cn
}

Received 26 April 2016; Revised 5 August 2016; Accepted 16 August 2016

Academic Editor: Konstantinos Demestichas

Copyright (C) 2016 Yiliang Chang et al. This is an open access article distributed under the Creative Commons Attribution License, which permits unrestricted use, distribution, and reproduction in any medium, provided the original work is properly cited.

Earlier works have studied the energy efficiency (EE) of half-duplex Device-to-Device (D2D) communications. However, the EE of full-duplex D2D communications underlaying cellular networks which undergoes residual self-interference (SI) has not been investigated. In this paper, we focus on the EE of full-duplex D2D communications with uplink channel reuse and compare it with the half-duplex counterpart, aiming to show which mode is more energy-efficient. Our goal is to find the optimal transmission powers to maximize the system EE while guaranteeing required signal-to-interference-plus-noise ratios (SINRs) and transmission power constraints. The optimal power allocation problem is modeled as a noncooperative game, in which each user equipment (UE) is self-interested and wants to maximize its own EE. An optimal iterative bisection-alternate optimization method is proposed to solve the optimization problem from the noncooperative game-theoretic perspective. Simulation results show that the proposed method can achieve EE close to that obtained by an existing method but with lower complexity in half-duplex D2D communications underlaying cellular networks. Moreover, the full-duplex D2D communications underlaying cellular networks outperform the halfduplex D2D communications underlaying cellular networks in terms of EE when effective SI mitigation techniques are applied.

\section{Introduction}

The exponential growth of wireless data services driven by mobile Internet and smart devices has triggered the investigation of the 5G cellular network. Around 2020, the new 5G mobile networks are expected to be deployed. 5G networks will have to support multimedia applications with a wide variety of requirements, including higher user data rates, reduced latency, enhanced coverage, and improved EE [1]. The primary techniques and approaches to address the requirements for $5 \mathrm{G}$ systems can be classified as in-band fullduplex, direct D2D communication, massive multiple-input multiple-output (massive MIMO), improved EE by energyaware communication and energy harvesting, and so forth [2-4]. Among these, the in-band full-duplex transmission, which allows transmitting and receiving at the same frequency and the same time, enables a significant enhancement of system throughput compared to half-duplex and has attracted a great deal of attention recently [5-7], whereas it suffers loop interference and requires effective SI mitigation [5].

On the other hand, D2D communications have been developed in recent years, which allow proximate cellular UE to communicate with each other directly under the control of base stations (BSs) with lower transmit power requirements. The high channel quality of short-range D2D links facilitates high data rates for local services, prolongs UE's battery lives, and offloads heavy traffic of BSs [8]. In addition, D2D links can underlay cellular links by reusing the same time and frequency resources, and thus the spectrum efficiency (SE) of cellular networks can be further improved. In the field of D2D communications, one of the key issues is the interference problem caused by resource sharing between D2D communications and traditional cellular communications, which 
has marked impacts on the performance of communication reliability and network throughput. Thus, effective power allocation is required to alleviate the interference problem.

D2D communications have already been designed as the application areas of small transmit power systems with short distance between communication devices. Considering the small transmit power requirements to reduce the effect of SI, low-power networks and short-range communications such as D2D are a potential scenario where full-duplex technique can be practically beneficial. This motivates us to apply fullduplex transmission into D2D communications, which is called full-duplex D2D communications. Implementing fullduplex transmission into D2D communications can efficiently increase the ergodic capacity of wireless cellular networks but will impose new challenges to D2D communications in wireless cellular networks. Since full-duplex transmission possesses strong SI, it generally consumes more power than half-duplex. Therefore, there is an urgent demand for maintaining high system throughput while limiting energy consumption [9]. EE, defined as the ratio of throughput to power consumption, is an important measure of green communication solutions [10]. The EE of full-duplex D2D communications underlaying cellular networks should be investigated. It is also interesting to compare the $\mathrm{EE}$ achieved by full-duplex D2D communications with its halfduplex counterpart.

1.1. Related Work. SI is a critical affecting factor of full-duplex communications. Consensus reached by both academia and industry showed that SI suppression/cancellation would play the most pivotal role in implementing radical full-duplex communication systems [6]. The authors in [11] provided a comprehensive overview on full-duplex D2D communications in heterogeneous networks, in which the potential solutions were proposed for interference mitigation. There are three main methods for SI mitigation: passive suppression (PS), analog cancellation (AC), and digital cancellation (DC) [5]. Among them, AC and DC both belong to active cancellation. Since the mitigation capability of traditional active cancellation techniques is very limited mainly due to the transmitter and receiver radio circuits' impairments, the PS will be a major role of SI cancellation [12]. PS is the first stage of SI cancellation, in which the SI signal is suppressed in the propagation domain before it is processed by the receiver circuitry. In addition to mitigating both the SI signal and noise in the propagation domain, it can also increase the dynamic range allocated for the desired signal before ADC which is an important performance limiting factor [5]. Traditional passive SI suppression usually benefits much from antenna separation, antenna isolation, directional antenna, or antenna polarization in some large-scale systems (e.g., relay systems) where the transmit and receive antennas are not collocated $[13,14]$. This large antenna separation usually requires a larger device size, but, in practical mobile applications with small device dimensions, the PS achieved by antenna separation and isolation is very limited. A more efficient passive SI suppression method with relatively small antenna separation was investigated in [15]. The results showed that a maximum of $72 \mathrm{~dB}$ PS can be achieved with different antenna separations ranging from 35 to $50 \mathrm{~cm}$ when absorptive shielding is present between the two antennas. But it is still not acceptable for mobile applications. Another more practical system in [16] with $10 \mathrm{~cm}$ antenna separation can obtain the average amount of $34 \mathrm{~dB}$ of PS, which is inefficient for full-duplex systems. Recently, Ahmed et al. in [12] proposed a novel full-duplex system utilizing multireconfigurable antenna (MRA) and the associated pattern selection algorithms for PS in mobile indoor applications. MRA is a dynamically reconfigurable antenna structure that is capable of changing its properties according to certain input configurations. Up to 16 different environments that include a variety of LOS, non-LOS, lowmobility, and high-mobility scenarios each with four different orientations (opposite, face-to-face, and two side-to-side orientations) were considered. The proposed approach can achieve an average of $65 \mathrm{~dB}$ PS at only $10 \mathrm{~cm}$ antenna separation without any antenna shielding. It is basically appropriate for mobile devices.

After PS, SI can be further mitigated by AC. The main idea is to obtain a copy of the transmitted radio frequency SI signal including all impairments and subtract it from the received signal in the analog domain. Requiring a higher cancellation capability implies more stringent requirements on the hardware. For example, to efficiently mitigate both main and reflected SI components at the receiver input, a multitap radio frequency finite impulse response (FIR) filter is used [17]. However, the size of the implemented radio frequency FIR filter limits the applicability of such technique. Furthermore, in the context of AC designs, such as the delayline-based technique in $[6,17]$, adopting more delay-line stages implies offering a higher delay resolution accuracy, however, attained at the cost of more complex and largesize hardware circuits. Thus, the applicability of AC on mobile devices may remain limited and in fact insufficient for hardware requirement $[6,17-19]$.

In addition to PS and AC, DC techniques can be performed in the digital domain, which are the lowest complexity active SI cancellation techniques in full-duplex systems [5, 19]. It is the last line of defense against SI, where the goal is to cancel the SI left over from the propagation domain and analog domain approaches. However, the SI cancellation amount achieved by conventional DC techniques is very limited, mainly due to hardware imperfections (e.g., phase noise, nonlinear distortion, and quantization noise) [18]. Recently, a novel digital SI cancellation technique based on new fullduplex transceiver architecture that significantly mitigates transmitter and receiver impairments was proposed in [19]. In the proposed technique, an auxiliary receiver chain is used to obtain a digital domain copy of the transmitted radio frequency SI signal, which is then used to cancel out the SI signal and the associated transmitter impairments in the digital domain. The proposed technique is shown to significantly mitigate the transmitter and receiver impairments without the necessity for highly complex analog domain cancellation techniques. The main advantage of the proposed technique is that all signal processing is performed in the digital domain, which significantly reduces the implementation complexity. The results show that the proposed technique significantly mitigates the SI signal up to $48 \mathrm{~dB}$ at $20 \mathrm{dBm}$ transmit power 
values. Besides, the overall full-duplex system performance using a combination of the proposed DC techniques and the practical PS techniques proposed in [12] can mitigate the SI signal to $3 \mathrm{~dB}$ higher than the receiver noise floor [19].

Since none of the individual cancellation techniques is capable of satisfying the system requirements in terms of the attainable SI cancellation capability, a high-capability cancellation scheme by combining the active and passive techniques is necessarily developed. Among all the SI cancellation techniques, the PS has an important position. The primary advantage of performing PS in the propagation domain is that the downstream receiver hardware can process signals more accurately with a large dynamic range [5]. As the design of AC usually requires complex and large-size hardware circuits with respect to mobile devices, a combination of the latest PS and DC (referred to as PSDC) techniques will be an appropriate way to perform the mobile full-duplex system $[12,19]$.

It is clear that deploying full-duplex transmission in current communication systems requires several architecture and protocol changes. The required changes depend on many factors, such as system type (e.g., relay system and pointto-point communication) and the deployed SI cancellation techniques. For the specific technique of point-to-point communication proposed in [12], some changes on the level of the physical and protocol layers are required. Although very few full-duplex realizations have been implemented in commercial mobile systems due to the technical and/or economic challenges, a substantial amount of related researches have already been undertaken in laboratory. The mobile fullduplex techniques will come into service in the near future.

Almost all previous researches on full-duplex D2D communications focused on improving throughput or SE [2025]. The EE of full-duplex D2D communications has not been investigated. Most of them ignore the significance of energy consumption of UE. In practical implementation, UE is typically handheld device with limited battery life and can quickly run out of battery if energy consumption is ignored in the system design. A power consumption model was presented in [26] for half-duplex D2D systems. It considered neither full-duplex mode nor static circuit power, which is actually comparable with the transmission power in short distance environments. For UE, its drain efficiency of power amplifier (PA) generally accounts for $38 \%$ of the total power [27]. Thus, the total power consumption mainly includes two parts in short distance environments: PA power and circuit power. In addition, applying active SI cancellation techniques consumes additional power; therefore, the power consumed by $\mathrm{AC}$ is nonnegligible. In $[28,29]$, the EE of half-duplex D2D communications without channel reuse was investigated. In [28], the authors analyzed the average EE of one-hop and multihop half-duplex D2D communications under Rayleigh fading channels, and the optimal UE transmission powers were derived to maximize EE. Sambo et al. in [29] presented the EE improvement with the deployment of half-duplex D2D communications in HetNets compared with the smallcell deployment. The work in $[30,31]$ presented the analysis of the EE of half-duplex D2D systems with cellular channel reuse. The work in [30] showed joint channel and power allocation to improve the EE of UE for half-duplex D2D communications underlaying cellular networks. In [31], the authors investigated the EE and SE in half-duplex D2D communications underlaying cellular networks with uplink channel reuse, where only each UE's EE is analyzed. In addition, an exhaustive EE analysis for both MIMO underlay and overlay D2D communications with a complete description of the mathematical framework was presented in [32]. To the best of our knowledge, an analysis of the EE of full-duplex D2D communications underlaying cellular networks is still lacking.

1.2. Summary of Contributions. In this paper, we provide a comprehensive EE analysis of full-duplex D2D communications underlaying cellular networks, in comparison to its halfduplex D2D counterpart. Our work is different from earlier works in the following aspects.

(i) This work investigates the EE of full-duplex D2D communications underlaying cellular networks and compares it with the half-duplex D2D counterpart. It is shown that, with given SINRs and transmission power constraints, the full-duplex D2D system can be more energy-efficient than the half-duplex D2D system.

(ii) The comparison of three modes, full-duplex (PSDC), full-duplex (PS), and half-duplex, where one mode outperforms another mode in terms of EE, is clearly presented. It is shown that full-duplex with PSDC can be more energy-efficient than full-duplex with PS as well as half-duplex, if the distance of the D2D pair is relatively close.

(iii) It is proved that each UE's EE is strictly quasiconcave with respect to its transmission power, under the maximum transmission power constraint. A noncooperative game-theoretic perspective is used to solve the power allocation problem to optimize the EE with the proposed low-complexity algorithm.

(iv) A full range of power consumption sources is considered in the power consumption model. In particular, the power consumed by SI cancellation is considered. The impact of imperfect SI cancellation is also considered in the analysis.

The rest of this paper is organized as follows. Section 2 introduces the system model for full-duplex and halfduplex D2D communications. The power allocation problem formulation for both communication modes is described in Section 3. In Section 4, the performance evaluation is presented. Finally, the conclusion is given in Section 5.

\section{System Model}

We consider a scenario of D2D communications underlaying a cellular network with cellular user equipment (CUE) and one D2D pair, in which the pair of D2D user equipment (DUE) pieces shares the same radio resources with the CUE in the uplink. In this paper, there are two different transmission modes for D2D communications: (a) full-duplex D2D 
communications and (b) half-duplex D2D communications. System models are presented separately for them.

\subsection{Mode A: Full-Duplex D2D Communications Underlaying} Cellular Networks. In the full-duplex D2D communications, the DUE operates in full-duplex mode (with one antenna dedicated to transmission and another to reception, in order to increase the isolation of the SI [16]) which can double the spectral resource utilization potentially, and the CUE operates in traditional cellular networks with a single antenna. The model of the full-duplex D2D system is illustrated in Figure 1, where DUE not only shares the uplink radio frequencytime resource with the CUE, but also transmits and receives simultaneously. Therefore, D2D transmissions will cause interference to the $\mathrm{BS}$ receiver, and $\mathrm{D} 2 \mathrm{D}$ receivers also suffer interference from the cellular uplink transmission. Moreover, the DUE in full-duplex mode transmits and receives at the same time and frequency, causing SI to the receivers from its transmitters. In order to analyze the effect of residual SI on system performance, we assume that the residual SI signal $v$ is zero mean, additive, and Gaussian, denoted as $v \sim$ $C N(0, V)[17,33-35]$. In addition, based on the experimental results in [16], the variance of the residual SI is modeled as $V=(1 / \beta) \bar{P}^{\lambda}$, where $\bar{P}$ is the transmission power of the $\mathrm{UE}$ and $\beta$ and $\lambda(0 \leq \lambda \leq 1)$ are constants that reflect the quality of the selected cancellation technique. This model generally includes two cases: the optimistic case in which interference variance is simply a constant and not a function of the transmission power $(\lambda=0)[17,36]$ and the other one in which the variance increases linearly with the transmission power $(\lambda=1)[33,34]$. We will choose the second case in which $\beta$ plays an important role in the EE of the full-duplex D2D communications.

To reduce the SI, PS and DC are applied. AC is not considered in this model due to high complexity and performance limitations on mobile applications (PS and DC can only be sufficiently effective for full-duplex transmission [19]). Similar to the previous work [16], let $\beta$ denote the total amount of SI cancellation at each DUE, which is defined as the ratio of the SI powers before and after cancellation. Thus, one gets $\beta=\beta_{\mathrm{PS}}$ in full-duplex (PS) and $\beta=\beta_{\mathrm{PS}}+\beta_{\mathrm{DC}}$ in full-duplex and half-duplex (PSDC). In general, $\beta_{\mathrm{PS}}$, which denotes the amount of SI cancelled by PS, can achieve up to $65 \mathrm{~dB}[12]$, and $\beta_{\mathrm{DC}}$, which denotes the amount of SI cancelled by $\mathrm{DC}$, can achieve $48 \mathrm{~dB}$ at most [19].

The received signal in the uplink at the BS is given by

$$
y_{\mathrm{bs}}=\sqrt{P_{u}} h_{u} x_{u}+\sum_{k=1}^{2} \sqrt{P_{d k}} h_{d k} x_{d k}+n_{\mathrm{bs}}, \quad k \in(1,2) \text {, }
$$

where $P_{u}$ is the transmission power of the CUE, $P_{d k}$ is the transmission power of $\mathrm{DUE}_{k}, h_{u}$ denotes the channel fading coefficient between the CUE and the BS, $h_{d k}$ denotes the channel fading coefficient between $\mathrm{DUE}_{k}$ and the BS, $x_{u}$ and $x_{d k}$ are the transmitted signals from the CUE and $\mathrm{DUE}_{k}$, respectively, and $n_{\mathrm{bs}}$ is an additive white Gaussian noise (AWGN) with zero mean and variance $\sigma^{2}$.
With residual SI, the received signal at $\mathrm{DUE}_{k}$ is given by

$$
\begin{aligned}
& y_{d k}=\sqrt{P_{d j}} h_{j k} x_{d j}+\sqrt{P_{u}} h_{u k} x_{u}+v_{d k}+n_{d k}, \\
& k, j \in\{1,2\}, k \neq j,
\end{aligned}
$$

where $h_{j k}$ denotes the channel fading coefficient between $\mathrm{DUE}_{j}$ and $\mathrm{DUE}_{k}, h_{u k}$ denotes the channel fading coefficient between the CUE and $\mathrm{DUE}_{k}$, and $n_{d k}$ is an AWGN with zero mean and variance $\sigma^{2}$. Additionally, $v_{d k}, k \in\{1,2\}$, is the residual SI signal at $\mathrm{DUE}_{k}$, which is expressed as $v_{d k} \sim \operatorname{CN}\left(0,\left(1 / \beta_{k}\right) P_{d k}\right), k \in(1,2)$, and $\beta_{1}, \beta_{2}$ depend on the amount of SI cancellation. The channel coefficients are assumed to be invariant in each transmission frame. The transmitted signals are zero mean and unit-variance.

The SINR of the full-duplex D2D mode at the BS and D2D receivers can be written as

$$
\begin{aligned}
& \gamma_{u}=\frac{P_{u}\left|h_{u}\right|^{2}}{\sum_{k=1}^{2} P_{d k}\left|h_{d k}\right|^{2}+\sigma^{2}}, \\
& \gamma_{d k}=\frac{P_{d j}\left|h_{j k}\right|^{2}}{P_{u}\left|h_{u k}\right|^{2}+1 / \beta_{k} \cdot P_{d k}+\sigma^{2}}, \\
& \quad k, j \in\{1,2\}, k \neq j,
\end{aligned}
$$

respectively. Then, the system SE of the full-duplex D2D mode is

$$
\begin{aligned}
\eta_{\mathrm{SE}, \mathrm{FD}}= & \mathrm{SE}_{u-\mathrm{FD}}+\mathrm{SE}_{d 1-\mathrm{FD}}+\mathrm{SE}_{d 2-\mathrm{FD}} \\
= & \log _{2}\left(1+\frac{P_{u}\left|h_{u}\right|^{2}}{\sum_{k=1}^{2} P_{d k}\left|h_{d k}\right|^{2}+\sigma^{2}}\right) \\
& +\log _{2}\left(1+\frac{P_{d 2}\left|h_{21}\right|^{2}}{P_{u}\left|h_{u 1}\right|^{2}+1 / \beta_{1} \cdot P_{d 1}+\sigma^{2}}\right) \\
& +\log _{2}\left(1+\frac{P_{d 1}\left|h_{12}\right|^{2}}{P_{u}\left|h_{u 2}\right|^{2}+1 / \beta_{2} \cdot P_{d 2}+\sigma^{2}}\right) .
\end{aligned}
$$

2.2. Mode B: Half-Duplex D2D Communications Underlaying Cellular Networks. In the half-duplex D2D communications, all of the UE pieces operate in half-duplex mode with a signal antenna, as shown in Figure 2. The overall process is completed in two successive slots $T_{1}$ and $T_{2}$ (the length of $T_{1}$ is equal to the length of $T_{2}$ ). DUE 1 transmits a signal to DUE in the first slot $T_{1}$ and $\mathrm{DUE}_{2}$ transmits a signal to $\mathrm{DUE}_{1}$ in the second slot, while the CUE transmits a signal with the same transmission power in two slots. By sharing the uplink radio resources with the CUE, the BS receives interference from D2D transmissions, and D2D receivers also get interference coming from the cellular uplink transmission.

In the first slot, the received signals at the $\mathrm{BS}$ and $\mathrm{DUE}_{2}$ are

$$
\begin{aligned}
\hat{y}_{\mathrm{bs} 1} & =\sqrt{\widehat{P}_{u}} \widehat{h}_{u} \widehat{x}_{u 1}+\sqrt{\widehat{P}_{d 1}} \widehat{h}_{d 1} \widehat{x}_{d 1}+\widehat{n}_{\mathrm{bs} 1}, \\
\hat{y}_{d 2} & =\sqrt{\widehat{P}_{d 1}} \widehat{h}_{12} \hat{x}_{d 1}+\sqrt{\widehat{P}_{u}} \widehat{h}_{u 2} \widehat{x}_{u 1}+\widehat{n}_{d 2},
\end{aligned}
$$

where $\widehat{P}_{u}$ is the transmission power of the CUE, $\widehat{P}_{d 1}$ is the transmission power of $\mathrm{DUE}_{1}, \widehat{h}_{u}$ denotes the channel fading 


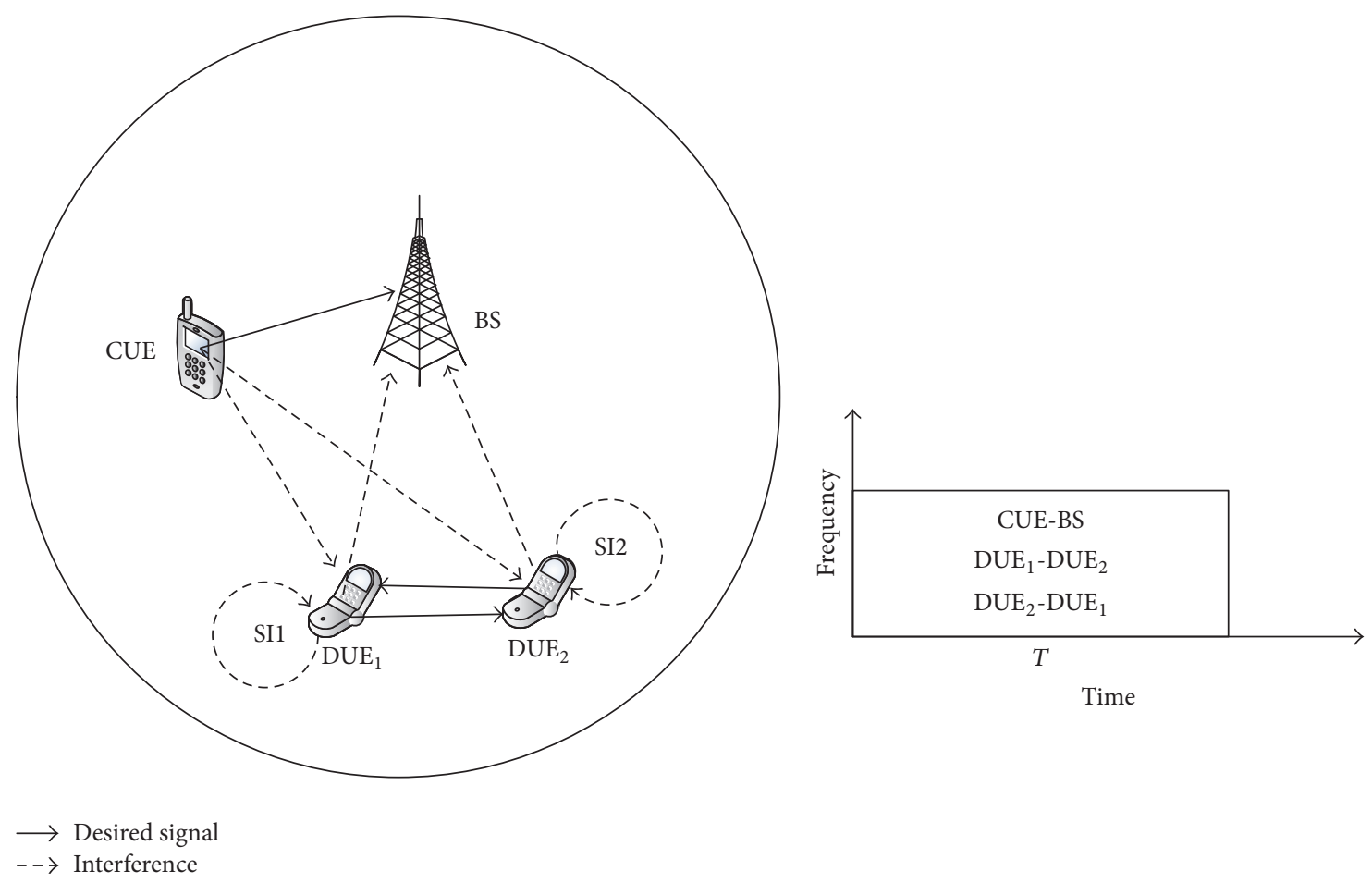

FIGURE 1: Full-duplex D2D communications underlaying cellular networks.

coefficient between the CUE and the BS, $\widehat{h}_{d 1}$ denotes the channel fading coefficient between $\mathrm{DUE}_{1}$ and the BS, $\widehat{h}_{12}$ denotes the channel fading coefficient between $\mathrm{DUE}_{1}$ and $\mathrm{DUE}_{2}, \widehat{h}_{u 2}$ denotes the channel fading coefficient between CUE and DUE ${ }_{2}, \widehat{n}_{\mathrm{bs} 1}$ and $\widehat{n}_{d 2}$ are AWGNs with zero mean and variance $\sigma^{2}$, and $\widehat{x}_{u 1}, \hat{x}_{d 1}$ are transmitted signals from the CUE and DUE ${ }_{1}$, respectively. are

In the second slot, the received signals at the $\mathrm{BS}$ and $\mathrm{DUE}_{1}$

$$
\begin{aligned}
\hat{y}_{\mathrm{bs} 2} & =\sqrt{\widehat{P}_{u}} \widehat{h}_{u} \widehat{x}_{u 2}+\sqrt{\widehat{P}_{d 2}} \widehat{h}_{d 2} \widehat{x}_{d 2}+\widehat{n}_{\mathrm{bs} 2}, \\
\hat{y}_{d 1} & =\sqrt{\widehat{P}_{d 2}} \widehat{h}_{21} \widehat{x}_{d 2}+\sqrt{\widehat{P}_{u}} \widehat{h}_{u 1} \widehat{x}_{u 2}+\widehat{n}_{d 1},
\end{aligned}
$$

where $\widehat{P}_{d 2}$ is the transmission power of DUE $2, \widehat{h}_{d 2}$ denotes the channel fading coefficient between $\mathrm{DUE}_{2}$ and the $\mathrm{BS}, \widehat{h}_{u 1}$ denotes the channel fading coefficient between the CUE and $\operatorname{DUE}_{1}, \widehat{h}_{21}$ denotes the channel fading coefficient between $\mathrm{DUE}_{2}$ and $\mathrm{DUE}_{1}, \widehat{n}_{\mathrm{bs} 2}$ and $\hat{n}_{d 1}$ are the AWGN with zero mean and variance $\sigma^{2}$, and $\widehat{x}_{u 2}, \widehat{x}_{d 2}$ are transmitted signals from the CUE and $\mathrm{DUE}_{2}$, respectively.

The SINR at the BS and D2D receivers can be written as

$$
\begin{aligned}
& \widehat{\gamma}_{u i}=\frac{\widehat{P}_{u}\left|\widehat{h}_{u}\right|^{2}}{\widehat{P}_{d i}\left|\widehat{h}_{d i}\right|^{2}+\sigma^{2}}, \quad i \in(1,2), \\
& \widehat{\gamma}_{d k}=\frac{\widehat{P}_{d j}\left|\widehat{h}_{j k}\right|^{2}}{\widehat{P}_{u}\left|\widehat{h}_{u k}\right|^{2}+\sigma^{2}}, \quad k, j \in\{1,2\}, \quad k \neq j,
\end{aligned}
$$

respectively. Then, the system SE of the half-duplex D2D mode is

$$
\begin{aligned}
\eta_{\mathrm{SE}, \mathrm{HD}}= & \mathrm{SE}_{u-\mathrm{HD}}+\mathrm{SE}_{d 1-\mathrm{HD}} \\
& +\mathrm{SE}_{d 2-\mathrm{HD}} \frac{1}{2} \sum_{i=1}^{2} \log _{2}\left(1+\frac{\widehat{P}_{u}\left|\widehat{h}_{u}\right|^{2}}{\widehat{P}_{d i}\left|\widehat{h}_{d i}\right|^{2}+\sigma^{2}}\right) \\
& +\frac{1}{2} \log _{2}\left(1+\frac{\widehat{P}_{d 2}\left|\widehat{h}_{21}\right|^{2}}{\widehat{P}_{u}\left|\widehat{h}_{u 1}\right|^{2}+\sigma^{2}}\right) \\
& +\frac{1}{2} \log _{2}\left(1+\frac{\widehat{P}_{d 1}\left|\widehat{h}_{12}\right|^{2}}{\widehat{P}_{u}\left|\widehat{h}_{u 2}\right|^{2}+\sigma^{2}}\right) .
\end{aligned}
$$

\section{Energy-Efficient Power Allocation}

In this section, we formulate the optimization problem for both the full-duplex and the half-duplex D2D modes which aims to maximize the system EE. Firstly, a novel definition of system EE based on summation of EE of all users rather than the ratio of sum network throughput to sum network power consumption was proposed [37]. Inspired of this novel definition, we define the system EE as the sum of the EE of DUE and CUE.

The EE of the whole system is defined as

$$
\eta_{\mathrm{EE}}=\sum_{n=1}^{N} \mathrm{EE}_{n}
$$

where $N$ denotes the number of users, $\mathrm{EE}_{n}$ which is expressed as the ratio of the $n$th user's data rate $r_{n}$ to the power 


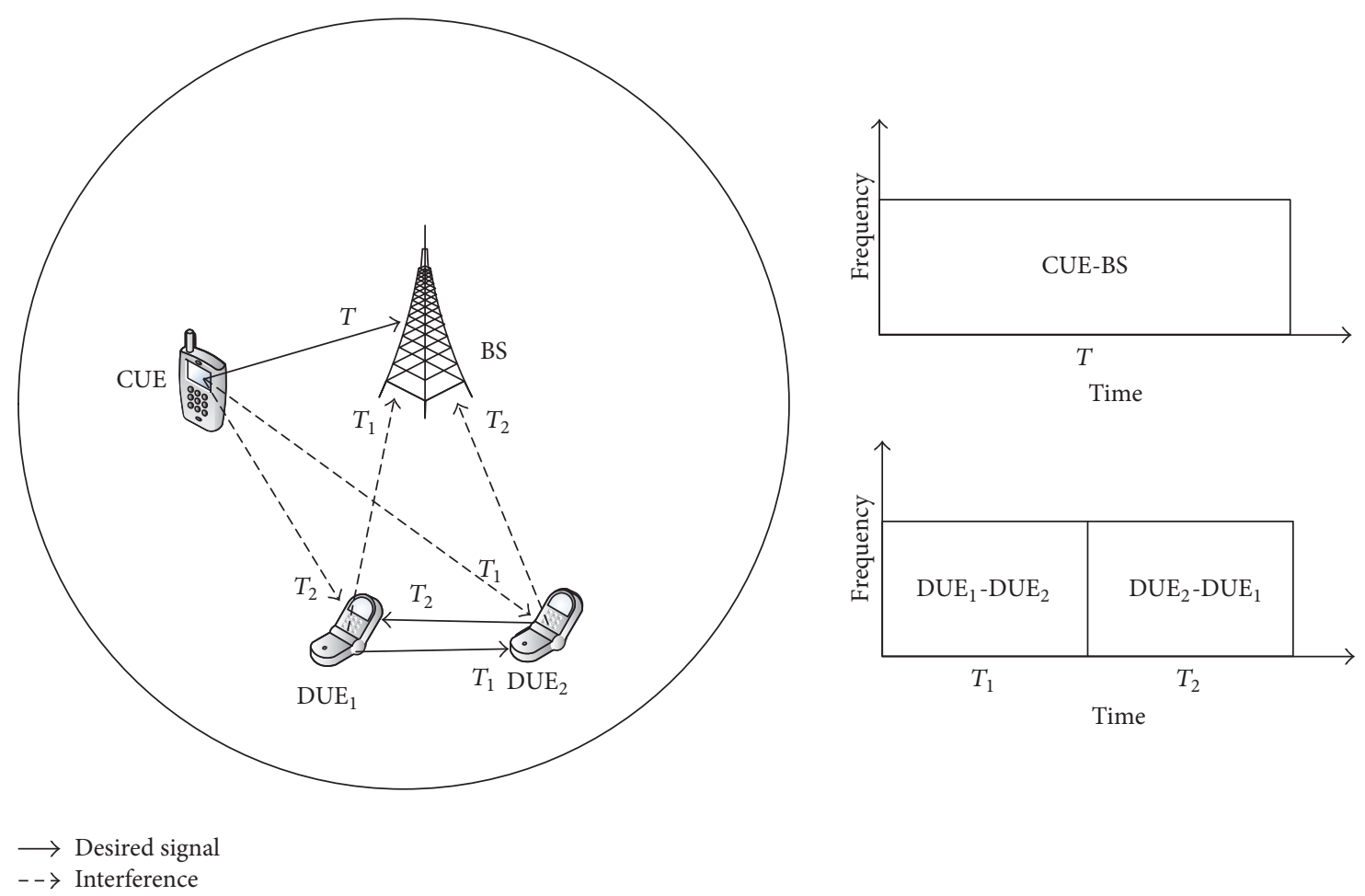

FIgURE 2: Half-duplex D2D communications underlaying cellular networks.

consumption $P_{n \text {-tot }}$ is the $\mathrm{EE}$ of $n$th user, and $\eta_{\mathrm{EE}}$ represents the total EE of all users. This definition is based on summation of EE of all users rather than the ratio of sum network throughout to sum network power consumption because powers of different users cannot be shared and so are their throughput and EE [37].

We need to determine power allocation of all users to optimize the overall network EE subject to the interference scenario. Note that the solution of maximum sum network EE is difficult to obtain as the objective function is in generally nonconcave in $P_{u}, P_{d 1}$, and $P_{d 2}$. More users in the system will result in more local maximum and searching the globally optimal power allocation would be difficult. Additionally, this mode is an interference-limited scenario in which the interference power is not easily known by the transmitters. Hence, a noncooperative game-theoretic perspective is adopted to solve the problem.

Rooted in economics, game theory has been broadly applied to several problems in wireless communications. It involves a set of competing players seeking for maximizing their own utility, based on certain actions they can take. When the users have no a priori knowledge about what others are simultaneously doing, the game is noncooperative, as users have no information on the basis of which they could cooperate [38]. Noncooperative game theory was first proposed as an efficient framework to formulate the power control problem for wireless data networks without centralized control. Accordingly, we model the power optimization from a noncooperative game-theoretic perspective and then investigate the existence of its equilibrium.
Combined with the general EE problem in (11), we consider the power allocation of user $n$ and denote the power vectors of other users by a vector

$$
P_{-n}=\left(P_{1}, P_{2}, \ldots, P_{n-1}, P_{n+1}, \ldots, P_{N}\right) \text {. }
$$

Then, the best response of the power allocation of user $n$ can be given by

$$
P_{n}^{*}=\arg \max _{P_{n}} \operatorname{EE}_{n}\left(P_{n}, P_{-n}\right),
$$

where $\mathrm{EE}_{n}$ is a function of both $P_{n}$ and $P_{-n}$ (existing in the form of interference).

Note that noncooperative power control is inefficient for SE optimization, because each user often behaves selfishly to increase their transmission powers which is far from generating a reasonable but quite small network SE [39]. To achieve a higher network SE, an efficient way is introducing pricing mechanisms to regulate the aggressive transmission powers of individuals to produce more socially beneficial outcome towards improving sum throughput of all users [38]. Different from SE-oriented optimal power control, energyefficient power optimization demands a power setting that is greedy in EE but chary of power. Furthermore, problem (13) is equivalent to

$$
\begin{aligned}
P_{n}^{*} & =\arg \max _{P_{n}} \log \left(\mathrm{EE}_{n}\left(P_{n}, P_{-n}\right)\right) \\
& =\arg \max _{P_{n}}\left(\log \left(r_{n}\right)-\log \left(P_{n \text {-tot }}\right)\right),
\end{aligned}
$$

which implies that energy-efficient power optimization can be regarded as a variation of traditional spectral-efficient 
one with power pricing [38]. Since this power-conservative expression is socially favorable in interference-limited scenarios, energy-efficient power optimization is desirable to reduce interference and improve throughput in the noncooperative setting [37].

In this noncooperative game, each user optimizes their transmission power independently. As the variation of transmission power of one user impacts those of all others, the strategies chosen by different users depend on each other and the achievement of a Nash equilibrium (i.e., the condition of a network in which the competing influences are balanced assuming invariant channel conditions) is a well-known optimality criterion to analyze the outcome of the game. Hence, we will characterize the equilibrium of noncooperative energy-efficient power optimization in the following subsections in detail.

The power consumption in a general wireless node for communications usually contains two mains parts, power amplifier and circuit power [31]. Power amplifier is related to the power amplifier drain efficiency and the transmission power level and is usually modeled as the ratio of the transmission power to the power amplifier drain efficiency [13]. Circuit power consumption is usually considered to be independent of the data rate and is regarded as a constant [40]. We consider both power amplifier and circuit power consumption in the transmitters. In addition, to be more practical, the circuit power consumption in the receivers is also considered. In a practical system, the circuit power consumption of BS in uplink is huge that can be supported by various efficient energy sources including renewable energy. Therefore, the EE of BS would be less critical as compared to that in the UE since mobile devices are typically handheld devices with limited battery life and can quickly run out of battery. So, it is reasonable to neglect the circuit power consumption of BS in uplink in the system EE [31, 41].

In full-duplex communications, applying PS generally does not consume additional power; however, the power consumed by active DC is nonnegligible. Besides, the power consumed by the involved chip components is not related to the throughput state, and therefore the power consumed by $\mathrm{DC}, P_{\mathrm{DC}}$, is regarded as a constant.

Thus, the power consumption for SI cancellation in fullduplex node can be expressed as $\omega P_{\mathrm{DC}}$, where $\omega=1$ for fullduplex and half-duplex (PSDC) transmission and $\omega=0$ for full-duplex (PS) transmission and half-duplex transmission. Each user has its own power range; that is, $0 \leq P_{u}\left(\widehat{P}_{u}\right) \leq P_{\max }$, $0 \leq P_{d 1}\left(\widehat{P}_{d 1}\right) \leq P_{\max }, 0 \leq P_{d 2}\left(\widehat{P}_{d 2}\right) \leq P_{\max }$, and $P_{\max }$ is the maximum allowed transmission power for the UE. The circuit power of different UE pieces is assumed to be the same, which is denoted as $P_{c}$.

3.1. Mode A: Full-Duplex D2D Communications Underlaying Cellular Networks. Based on the novel definition above, the system EE of the full-duplex D2D mode can be written as

$$
\begin{aligned}
\eta_{\mathrm{EE}-\mathrm{FD}} & =\eta_{u-\mathrm{FD}}+\eta_{d 1-\mathrm{FD}}+\eta_{d 2-\mathrm{FD}} \\
& =\frac{W \cdot \mathrm{SE}_{u-\mathrm{FD}}}{\xi_{u} P_{u}+P_{c}}+\frac{W \cdot \mathrm{SE}_{d 1-\mathrm{FD}}}{\xi_{d} P_{d 2}+P_{c}+\omega P_{\mathrm{DC}}}
\end{aligned}
$$

$$
+\frac{W \cdot \mathrm{SE}_{d 2-\mathrm{FD}}}{\xi_{d} P_{d 1}+P_{c}+\omega P_{\mathrm{DC}}},
$$

where $W$ denotes the channel bandwidth and $1 / \xi_{u}$ and $1 / \xi_{d}$ are the power amplifier drain efficiency of the CUE and DUE, respectively. Our objective is to find the optimal transmission powers $P_{u}, P_{d 1}$, and $P_{d 2}$ to maximize $\eta_{\mathrm{EE}-\mathrm{FD}}$ while keeping $\gamma_{u}, \gamma_{d k}$, and $k \in\{1,2\}$ above the thresholds and satisfying the maximum power constraints of the UE, which can be formulated as

$$
\begin{aligned}
\max _{\left\{P_{u}, P_{d 1}, P_{d 2}\right\}} & \eta_{\mathrm{EE}-\mathrm{FD}} \\
\text { s.t. } & \gamma_{u} \geq \bar{\gamma}_{u}, \\
& \gamma_{d 1} \geq \bar{\gamma}_{d}, \\
& \gamma_{d 2} \geq \bar{\gamma}_{d}, \\
& 0 \leq P_{u} \leq P_{\max }, \\
& 0 \leq P_{d 1} \leq P_{\max }, \\
& 0 \leq P_{d 2} \leq P_{\max },
\end{aligned}
$$

where $\bar{\gamma}_{u}, \bar{\gamma}_{d}$ are the minimum required SINRs of the CUE and DUE, respectively, and $P_{\max }$ is the maximum allowed transmission power of all users.

Unfortunately, joint optimization over $P_{u}, P_{d 1}$, and $P_{d 2}$ is very hard to be found due to the idea that $\eta_{\mathrm{EE}-\mathrm{FD}}$ is not concave in $P_{u}, P_{d 1}$, and $P_{d 2}$ jointly. So it cannot be solved by the general convex optimization methods. Though the Dinkelbach method can be used to solve the optimization problem, its computational complexity may be high. Therefore, the bisection method is used to solve the optimization problem with lower complexity but close performance to the Dinkelbach method. According to the analysis in the beginning of Section 3, the EE model is an interference-limited scenario in which the transmission powers $P_{u}, P_{d 1}$, and $P_{d 2}$ are not independent and the interference power is not easily known by the transmitters; thus, a noncooperative gametheoretic perspective is used to solve problem (16). In the noncooperative game, the achievement of a Nash equilibrium is a well-known optimality criterion to analyze the outcome of the game. In this paper, a set of power allocations is said to be at Nash equilibrium if no user can improve its EE individually by unilaterally altering its own transmission power. To find the Nash equilibrium of our noncooperative optimization problem, we first address the properties of a defined function $f(a, p)=\log _{2}(1+a p) /\left(\xi p+p_{c}\right)$, where $p$ denotes the transmission power, $1 / \xi$ denotes the power amplifier drain efficiency, and $a$ is an expression derived from the expression of EE. According to the definition of quasiconcavity in [37], the quasiconcavity of $f(a, p)$ is proved in the following Theorem 1. Additionally, the SI power contained in $a$ has no effect on quasiconcavity of $f(a, p)$.

Theorem 1. The function $f(a, p)$ is strictly quasiconcave in $p$ for $p \in[0,+\infty)$.

Proof. Refer to Appendix A. 


$$
\begin{aligned}
& \text { Input: given } p \leq p^{*} \leq \bar{p} \text {, and } \varepsilon>0 \\
& \text { Output: the optimal } p^{*} \\
& \text { (1) Repeat } \\
& \text { (2) } m=(\underline{p}+\bar{p}) / 2 \\
& \text { (3) } \Delta=\left.(\bar{\partial} f(a, p) / \partial p)\right|_{p=m} \\
& \text { (4) if } \Delta>0 \\
& \text { (5) } \underline{p}=m \\
& \text { (6) else } \\
& \text { (7) } \bar{p}=m \\
& \text { (8) end if } \\
& \text { (9) until } \bar{p}-\underline{p} \leq \varepsilon \\
& \text { (10) } p^{*}=(\overline{\bar{p}}+\underline{p}) / 2
\end{aligned}
$$

Algorithm 1: Bisection method for quasiconcave optimization.

Obviously, $\eta_{u \text {-FD }}, \eta_{d 1-\mathrm{FD}}$, and $\eta_{d 2 \text {-FD }}$ are strictly quasiconcave in $P_{u}, P_{d 1}$, and $P_{d 2}$, respectively. Based on Theorem 1, the existence of the Nash equilibrium can be proved in Theorem 2 .
Theorem 2. The Nash equilibrium exists in the noncooperative power optimization game with a set of power allocations of $P_{u}$, $P_{d 2}$, and $P_{d 1}$.

Proof. Refer to Appendix B.

In the formulated noncooperative full-duplex EE maximization problem, each user optimizes their power independently. The best response of one transmitter power depends on the transmitter powers of all other UE pieces which cannot be obtained in the noncooperative setting. However, we observe that the sufficient information of other users' transmission powers is contained in the form of interference. In this way, each DUE only has to estimate the interference on the shared channel to determine the power optimization rather than knowing the specific transmitter powers of other UE pieces. For the CUE, the BS estimates the interference from $\mathrm{D} 2 \mathrm{D}$ pair on the shared channel and then feeds back this information to the CUE. Besides, the SI of DUE in the fullduplex mode can be got depending on its own transmission powers and SI cancellation techniques.

Hence, at the time instant $t+1$, the predicted EE of CUE, $\mathrm{DUE}_{1}$, and $\mathrm{DUE}_{2}$ in the full-duplex system is

$$
\begin{aligned}
\eta_{u-\mathrm{FD}}(t+1) & =\frac{W \log _{2}\left(1+P_{u}(t+1)\left|h_{u}\right|^{2} /\left(I_{u-\mathrm{FD}}(t)+\sigma^{2}\right)\right)}{\xi_{u} P_{u}(t+1)+P_{c}}, \\
\eta_{d 1-\mathrm{FD}}(t+1) & =\frac{W \log _{2}\left(1+P_{d 2}(t+1)\left|h_{21}\right|^{2} /\left(I_{d 1-\mathrm{FD}}(t)+1 / \beta \cdot P_{d 1}(t)+\sigma^{2}\right)\right)}{\xi_{d} P_{d 2}(t+1)+P_{c}+\omega P_{\mathrm{DC}}}, \\
\eta_{d 2-\mathrm{FD}}(t+1) & =\frac{W \log _{2}\left(1+P_{d 1}(t+1)\left|h_{12}\right|^{2} /\left(I_{d 2-\mathrm{FD}}(t)+1 / \beta \cdot P_{d 2}(t)+\sigma^{2}\right)\right)}{\xi_{d} P_{d 1}(t+1)+P_{c}+\omega P_{\mathrm{DC}}},
\end{aligned}
$$

respectively, where $I_{u \text {-FD }}(t)$ is the measured interference power of CUE from DUE ${ }_{1}$ and $\mathrm{DUE}_{2}$ at the time instant $t$ and $I_{d 1-\mathrm{FD}}(t)$ and $I_{d 2-\mathrm{FD}}(t)$ are the measured interference powers of $\mathrm{DUE}_{1}$ and $\mathrm{DUE}_{2}$ from CUE at the time instant $t$, respectively.

Due to the strict quasiconcavity of $\eta_{u-\mathrm{FD}}, \eta_{d 1-\mathrm{FD}}$, and $\eta_{d 2-\mathrm{FD}}$, numerical methods like the bisection method can be used to find the optimal power allocation at each time slot. For a more concise analysis, the optimal solution of the above-mentioned general function $f(a, p)$ with the bisection method is shown in Algorithm 1.

In Algorithm 1, $p$ is the lower bound and $\bar{p}$ is the upper bound of the optimal power $p^{*}$. The optimization of $f(a, p)$ starts with an interval $[p, \bar{p}]$ that contains the optimal value $p^{*}$ and concludes the optimal value of $f(a, p)$.

Clearly, Algorithm 1 can generate the optimal solutions of (17)-(19), respectively. Then, we introduce an iterative bisection-alternate optimization method to search for the optimal power allocation. The basic idea of the method is to search a better power allocation of all users alternately with the bisection method at each time slot and enable iterative search for a long time until the EE convergence of the fullduplex system. The iterative bisection-alternate optimization method of the full-duplex D2D communications is described in Algorithm 2.

3.2. Mode B: Half-Duplex D2D Communications Underlaying Cellular Networks. In the half-duplex D2D mode, the system EE can be written as

$$
\begin{aligned}
\eta_{\mathrm{EE}-\mathrm{HD}}= & \eta_{u-\mathrm{HD}}+\eta_{d 1-\mathrm{HD}}+\eta_{d 2-\mathrm{HD}} \\
= & \frac{W \cdot \mathrm{SE}_{u-\mathrm{HD}}}{\xi_{u} \widehat{P}_{u}+P_{c}}+\frac{W \cdot \mathrm{SE}_{d 1-\mathrm{HD}}}{(1 / 2) \xi_{d} \widehat{P}_{d 2}+P_{c}} \\
& +\frac{W \cdot \mathrm{SE}_{d 2-\mathrm{HD}}}{(1 / 2) \xi_{d} \widehat{P}_{d 1}+P_{c}},
\end{aligned}
$$

where the factor $1 / 2$ indicates that the transmitters send data only during half of the entire frame (during $T_{1}$ and $T_{2}$, resp.) and thus consume power only during half of the entire frame. For DUE, the circuit power $P_{c}=P_{c}^{T}+P_{c}^{R}$, where $P_{c}^{T}$ and $P_{c}^{R}$ denote the circuit consumption during half of the entire frame in the transmitter and receiver, respectively. For CUE, we consider the circuit power only at the transmitter side during the entire frame, that is, $P_{c}$ [31]. Similar to the fullduplex D2D mode, our objective is to find the optimal 


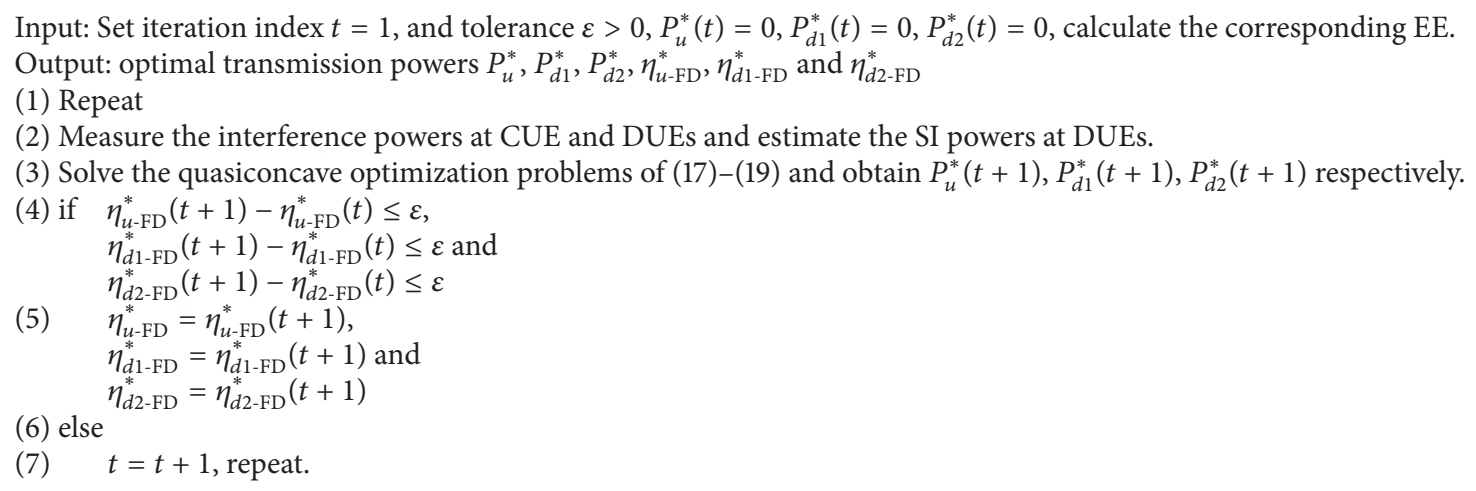

Algorithm 2: Iterative bisection-alternate optimization method for full-duplex D2D.

transmission powers $\widehat{P}_{u}, \widehat{P}_{d 1}$, and $\widehat{P}_{d 2}$ to maximize $\eta_{\text {EE-HD }}$ while keeping $\widehat{\gamma}_{u i}, k \in\{1,2\}$, and $\widehat{\gamma}_{d k}, k \in\{1,2\}$ above the thresholds and satisfying the maximum power constraints of the UE; that is,

$$
\begin{aligned}
\max _{\left\{\widehat{P}_{u}, \widehat{P}_{d 1} \widehat{P}_{d 2}\right\}} & \eta_{\text {EE-HD }} \\
\text { s.t. } & \widehat{\gamma}_{u 1} \geq \bar{\gamma}_{u}, \\
& \widehat{\gamma}_{u 2} \geq \bar{\gamma}_{u}, \\
& \widehat{\gamma}_{d 1} \geq \bar{\gamma}_{d}, \\
& \widehat{\gamma}_{d 2} \geq \bar{\gamma}_{d}, \\
& 0 \leq \widehat{P}_{u} \leq P_{\max }, \\
& 0 \leq \widehat{P}_{d 1} \leq P_{\max }, \\
& 0 \leq \widehat{P}_{d 2} \leq P_{\max } .
\end{aligned}
$$

$$
\begin{aligned}
\eta_{u-\mathrm{HD}}(t+1) & =\frac{(W / 2)\left\{\log _{2}\left(1+\widehat{P}_{u}(t+1)\left|\widehat{h}_{u}\right|^{2} /\left(I_{u 1-\mathrm{HD}}(t)+\sigma^{2}\right)\right)+\log _{2}\left(1+\widehat{P}_{u}(t+1)\left|\widehat{h}_{u}\right|^{2} /\left(I_{u 2-\mathrm{HD}}(t)+\sigma^{2}\right)\right)\right\}}{\xi_{u} \widehat{P}_{u}(t+1)+P_{c}} \\
\eta_{d 1-\mathrm{HD}}(t+1) & =\frac{(W / 2) \log _{2}\left(1+\widehat{P}_{d 2}(t+1)\left|\widehat{h}_{21}\right|^{2} /\left(I_{d 1-\mathrm{HD}}(t)+\sigma^{2}\right)\right)}{(1 / 2) \xi_{d} \widehat{P}_{d 2}(t+1)+P_{c}} \\
\eta_{d 2-\mathrm{HD}}(t+1) & =\frac{(W / 2) \log _{2}\left(1+\widehat{P}_{d 1}(t+1)\left|\widehat{h}_{12}\right|^{2} /\left(I_{d 2-\mathrm{HD}}(t)+\sigma^{2}\right)\right)}{(1 / 2) \xi_{d} \widehat{P}_{d 1}(t+1)+P_{c}}
\end{aligned}
$$

respectively, where $I_{u 1-\mathrm{HD}}(t)$ and $I_{u 2-\mathrm{HD}}(t)$ are the measured interference powers of CUE from $\mathrm{DUE}_{1}$ and $\mathrm{DUE}_{2}$ at the time instant $t$, respectively, and $I_{d 1-\mathrm{HD}}(t)$ and $I_{d 2-\mathrm{HD}}(t)$ are the measured interference powers of DUE ${ }_{1}$ and $D E_{2}$ from CUE at the time instant $t$, respectively.

The solutions of quasiconcave problems (22)-(24) at each time slot can be solved by numerical methods like the bisection method, respectively. Then, we use the iterative bisection-alternate optimization method to search for the optimal power allocation. The basic idea of the method is to search a better power allocation of all users alternately with the bisection method at each time slot and enable iterative search for a long time until the EE convergence of the halfduplex system. The iterative bisection-alternate optimization 
Input: Set iteration index $t=1$, and tolerance $\varepsilon>0, \widehat{P}_{u}^{*}(t)=0, \widehat{P}_{d 1}^{*}(t)=0, \widehat{P}_{d 2}^{*}(t)=0$, calculate the corresponding EE. Output: optimal transmission powers $\widehat{P}_{u}^{*}, \widehat{P}_{d 1}^{*}, \widehat{P}_{d 2}^{*}, \eta_{u-\mathrm{HD}}^{*}, \eta_{d 1-\mathrm{HD}}^{*}$ and $\eta_{d 2-\mathrm{HD}}^{*}$

(1) Repeat

(2) Measure the interference powers at CUE and DUEs.

(3) Solve the quasiconcave optimization problems of (22)-(24) and obtain $P_{u}^{*}(t+1), P_{d 1}^{*}(t+1), P_{d 2}^{*}(t+1)$ respectively.

(4) if $\eta_{u-\mathrm{HD}}^{*}(t+1)-\eta_{u-\mathrm{HD}}^{*}(t) \leq \varepsilon$,

$\eta_{d 1-\mathrm{HD}}^{*}(t+1)-\eta_{d 1-\mathrm{HD}}^{*}(t) \leq \varepsilon$ and

$\eta_{d 2-\mathrm{HD}}^{*}(t+1)-\eta_{d 2-\mathrm{HD}}^{*}(t) \leq \varepsilon$

(5) $\eta_{u-\mathrm{HD}}^{*}=\eta_{u-\mathrm{HD}}^{*}(t+1)$,

$\eta_{d 1-\mathrm{HD}}^{*}=\eta_{d 1-\mathrm{HD}}^{*}(t+1)$ and

(6) else

$\eta_{d 2-\mathrm{HD}}^{*}=\eta_{d 2-\mathrm{HD}}^{*}(t+1)$

(7) $t=t+1$, repeat.

Algorithm 3: Iterative bisection-alternate optimization method for half-duplex D2D.

method of the half-duplex D2D communications is described in Algorithm 3.

\section{Simulation Results}

In this section, the effectiveness of the proposed algorithms is verified by using the MATLAB. The bandwidth $W$ is normalized to 1 ; that is, $W=1 \mathrm{~Hz}$. The maximum transmission power constraints of the CUE and DUEs are set to $P_{\max }=1 \mathrm{~W}$ and the circuit power consumption is set to $P_{c}=100 \mathrm{~mW}$. The power consumption coefficients of CUE and DUE are set to $38 \%$ [27]; that is, $\xi_{u}=\xi_{d}=1 / 0.38$. The noise power is normalized to 1 ; that is, $\sigma^{2}=1 \mathrm{~W}$. The minimum SINR thresholds $\bar{\gamma}_{u}$ and $\bar{\gamma}_{d}$ are set to $0 \mathrm{~dB}$.

Firstly, we compare the EE and complexity of the proposed method with the Lagrangian method in the existing literature [31] for the half-duplex D2D communications underlaying cellular networks, and then we show the convergence of our proposed algorithm.

In order to increase comparability with the existing method in [31], we show the EE and SE tradeoff and the execution time for half-duplex D2D communications obtained by our proposed method and the Lagrangian method in Figure 3 , in which we assume that all useful channel coefficients are set to $5 \mathrm{~dB}$ and all interference channel coefficients are set to $0 \mathrm{~dB}$. For each method, with the increase of the $\mathrm{SE}$ requirement, the corresponding EE is obtained. Simulation results show that the two methods can achieve EE close to each other under the same conditions. Besides, the figure also shows that our proposed method gives faster time execution than the Lagrangian method. Consequently, the performance of our proposed method is consistent with the Lagrangian method but with lower complexity.

Figure 4 shows the convergence speed of the bisectionalternate method for the system EE and total power consumption under full-duplex and half-duplex D2D modes, respectively, where the total amount of SI cancellation of fullduplex is set to $\beta_{1}=\beta_{2}=60 \mathrm{~dB}$, the useful channel coefficient of CUE is set to $4 \mathrm{~dB}$, the useful channel coefficient of DUE is set to $8 \mathrm{~dB}$, and all interference channel coefficients are set to $0 \mathrm{~dB}$. For comparison, the exhaustive searching method is also used to show the convergence speed which provides the maximum EE and optimal power. From this figure, one can see that the results obtained by the proposed method and the exhaustive searching method are exactly the same, which shows that the proposed method can find the optimal solution. It also can be seen that all of the curves converge to stable values finally. It is obvious that the full-duplex D2D mode can increase the system EE as compared with the half-duplex D2D mode although the full-duplex D2D mode suffers higher power consumption due to SI.

Secondly, we focus on the effect of distance and total amount of SI cancellation on system performance. We consider a single circular cell on the two-dimensional $X-Y$ plane and the radius is normalized to $1 \mathrm{~m}$. The BS is located at the center with coordinate $(0,0)$. The distance between $\mathrm{DUE}_{1}$ and $\mathrm{DUE}_{2}$ is $d_{D}$, and the distance between BS and CUE is $d_{\mathrm{CUE}, \mathrm{BS}}$. We assume that the coordinates of CUE, DUE ${ }_{1}$, and DUE 2 are $(-0.08,0),(-0.04,-0.8)$, and $(0.04,-0.8)$, respectively. Note that the transmit antenna is very close to the receive antenna for $\mathrm{D} 2 \mathrm{D}$ users. The reasonable distance between the transmit and receive antennas used for antenna separation is about $10 \mathrm{~cm}$. In addition, all of the channel fading coefficients are modeled as circularly symmetric complex Gaussian random variables with zero mean and variances $d^{-\alpha}$, where $d$ denotes the distance between any two nodes and $\alpha$ denotes the path loss exponent, which is set to 4 .

In Figure 5, the system EE versus the distance between $\mathrm{DUE}_{1}$ and $\mathrm{DUE}_{2}$ is illustrated. The total SI cancellation amounts of FD (PS) and FD (PSDC) are set to $\beta_{1}=\beta_{2}=$ $60 \mathrm{~dB}$ and $90 \mathrm{~dB}$, respectively, which are within the reasonable range of cancellation amount as discussed in Sections 1.1 and 2. Since D2D communications are mainly applied in short distance situation, the distance between $\mathrm{DUE}_{1}$ and $\mathrm{DUE}_{2}$ is assumed in the range from 0.08 to 0.4 , which corresponds to the $X$-coordinates of $\mathrm{DUE}_{2}$ from 0.04 to 0.36 . When the $X$ coordinates of the $\mathrm{DUE}_{2}$ change, the distance between $\mathrm{DUE}_{2}$ and the CUE (BS) also changes; that is, the corresponding interference also changes. From this figure, as the distance of D2D pair increases, one can see that the system EE of both modes decreases. As expected, with better SI cancellation, full-duplex (PSDC) achieves higher EE than full-duplex (PS). 

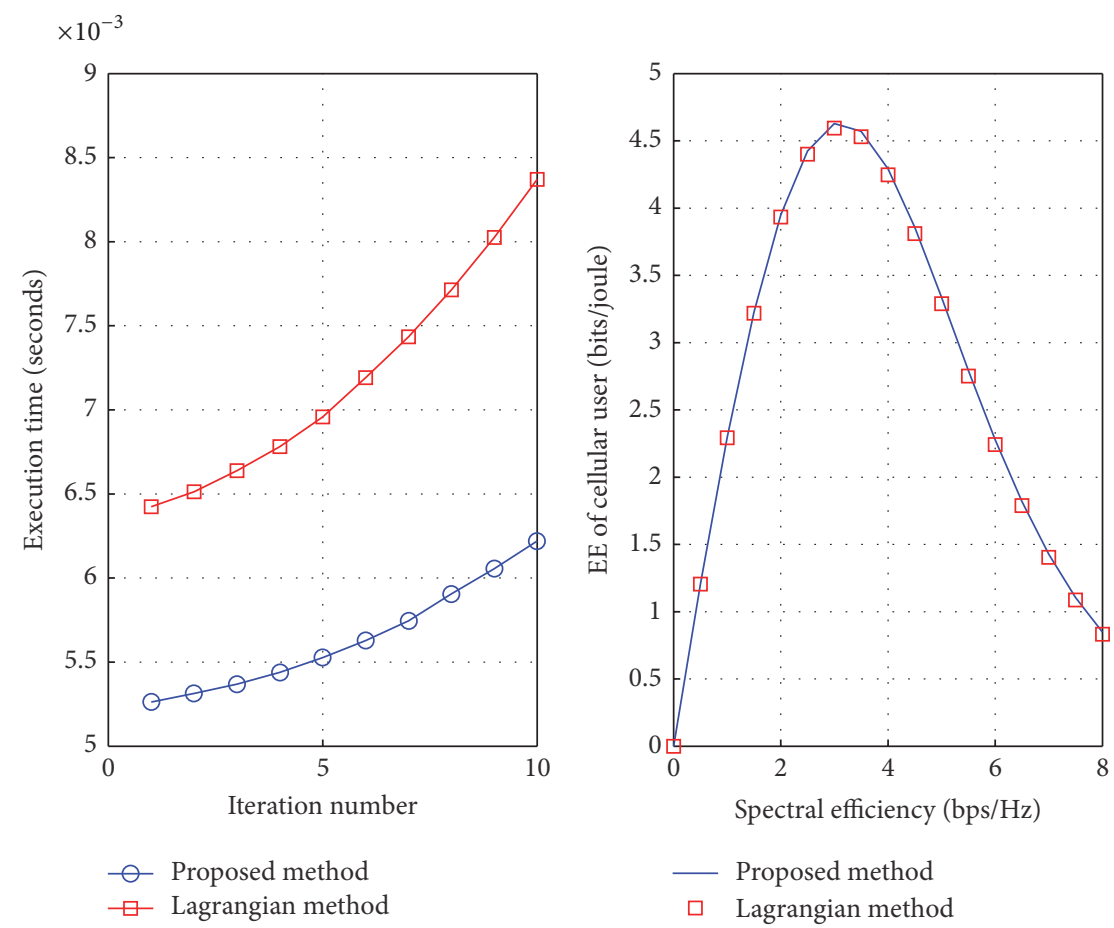

FIGURE 3: Comparison between the proposed method and the Lagrangian method: time of execution and the EE and SE tradeoff for the cellular link.

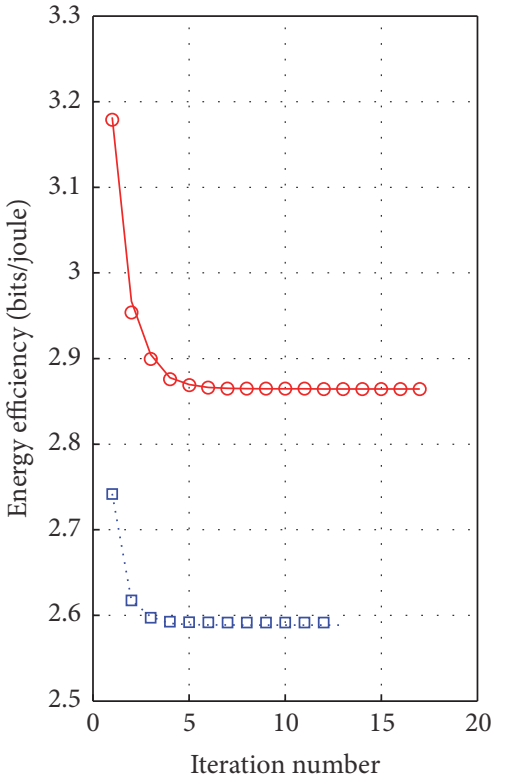

- FD-exhaustive 0 FD-bisection HD-exhaustive a HD-bisection

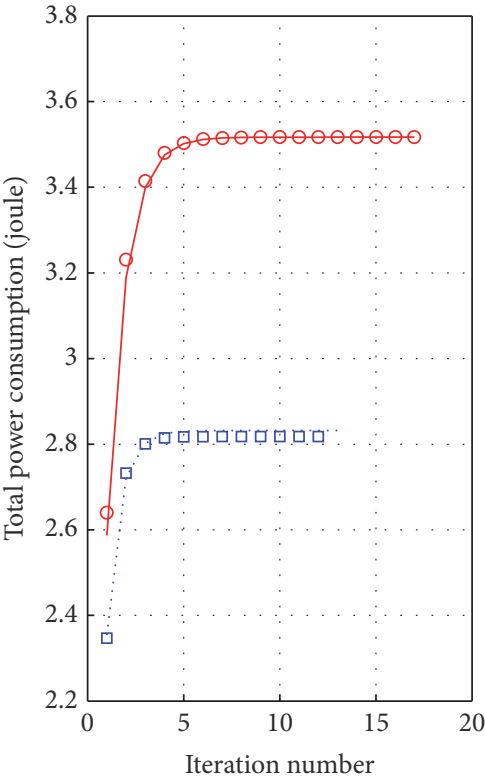

- FD-exhaustive $\circ$ FD-bisection ..... HD-exhaustive a HD-bisection

FIGURE 4: The system EE and total power consumption of full-duplex D2D mode and half-duplex D2D mode versus number of iterations by the bisection-alternate method and the exhaustive searching method.

Moreover, both full-duplex D2D modes have higher system EE than the half-duplex D2D mode with a short distance of D2D pair, which indicates that the full-duplex D2D mode outperforms the half-duplex D2D mode in the short distance transmission of D2D. As the distance increases, this situation is reversed. This is because the transmission power of fullduplex D2D mode increases vastly in order to combat the residual SI as the distance of D2D pair increases.

The system EE versus distance between the CUE and the BS is illustrated in Figure 6, where the total amounts of 


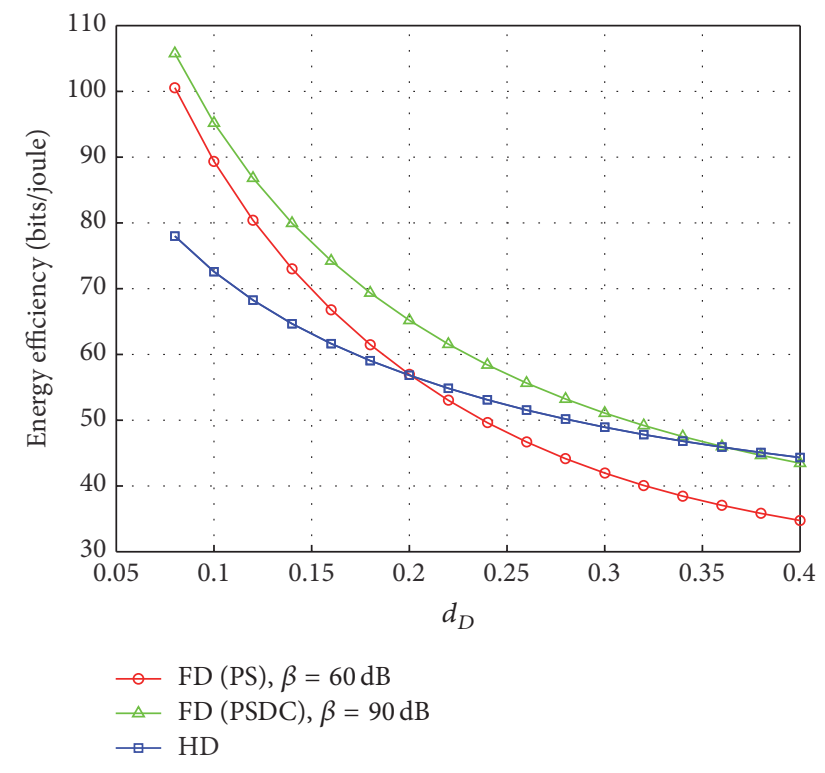

Figure 5: The system EE of full-duplex D2D mode and half-duplex $\mathrm{D} 2 \mathrm{D}$ mode versus distance of $\mathrm{D} 2 \mathrm{D}$ pair.

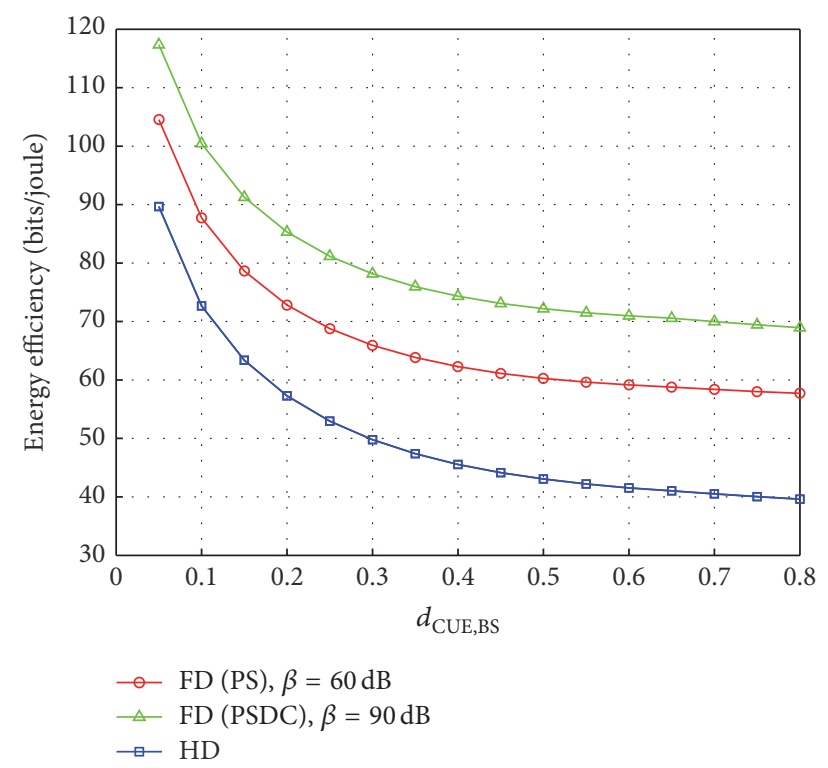

FIGURE 6: The system EE of full-duplex D2D mode and half-duplex D2D mode versus distance between CUE and BS.

SI cancellation of full-duplex are set to $\beta_{1}=\beta_{2}=60 \mathrm{~dB}$ (PS) and $90 \mathrm{~dB}$ (PSDC), respectively. It is assumed that the distance between the CUE and the BS ranges from 0.05 to 0.8 , which corresponds to the $X$-coordinates of the CUE from -0.05 to -0.8 . When the distance between the CUE and the BS changes, the distance between the CUE and D2D pair also changes. One can see that the system EE of the two full-duplex D2D modes is still better than that of the halfduplex D2D mode because of higher spectrum utilization efficiency. Moreover, full-duplex (PSDC) with large amount of SI cancellation can achieve higher EE than full-duplex (PS). We can also observe that the system EE decreases when the

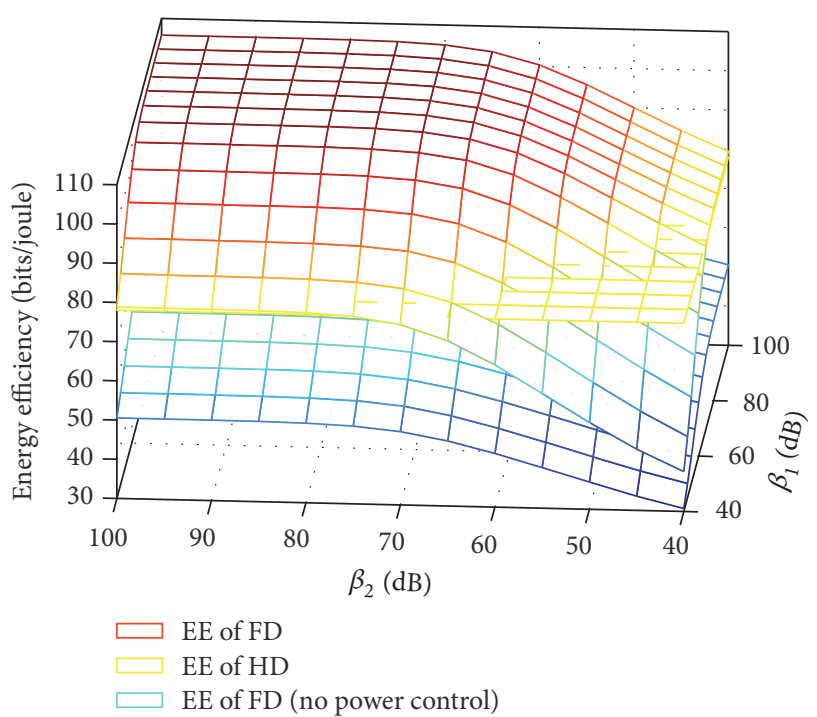

FIGURE 7: The system EE comparison of the half-duplex D2D mode and full-duplex D2D mode with residual SI.

distance between the CUE and the BS increases. In this case, the position of the cellular user has an overwhelming effect on the system EE.

Figure 7 compares the system EE among the full-duplex and half-duplex D2D mode with power control and the fullduplex D2D mode without power control under different amounts of SI cancellation of the D2D pair. As $\beta_{1}$ and $\beta_{2}$ increase, indicating that the amounts of SI cancellation increase, the system EE obtained by the full-duplex D2D mode increases. There is a cross line between the full-duplex and half-duplex surfaces, indicating that if the residual SI is mitigated to some extent, the full-duplex D2D mode can achieve higher system EE than the half-duplex D2D mode. We can also observe that the full-duplex D2D mode without power control has the lowest EE. In practical full-duplex communication scenarios with PS and DC techniques, the EE enhancement achieved by full-duplex (PS, $\beta_{1}=\beta_{2}=60 \mathrm{~dB}$ ) and full-duplex and half-duplex (PSDC, $\beta_{1}=\beta_{2}=90 \mathrm{~dB}$ ) are $19 \%$ and $36 \%$ compared to the half-duplex counterpart. Besides, the EE enhancement achieved by full-duplex (PS, $\beta_{1}=\beta_{2}=60 \mathrm{~dB}$ ) and full-duplex (PSDC, $\beta_{1}=\beta_{2}=90 \mathrm{~dB}$ ) is $64 \%$ and $51 \%$ compared to no power control counterpart.

\section{Conclusions}

In this paper, the issue of system EE maximization of fullduplex D2D communications in cellular networks while a D2D pair shares the uplink resources with CUE has been investigated. With a full range of power consumption sources considered, we compared the EE of three modes: full-duplex (PSDC), full-duplex (PS), and half-duplex counterpart. Such an issue was formulated as a nonconvex optimization problem in which the objective is to maximize the system EE while satisfying required SINR and transmission power constraints. From the noncooperative game-theoretic perspective, the iterative bisection-alternate method with lower complexity 
was proposed to solve the optimization problem. It is found that full-duplex (PS) can outperform half-duplex in terms of EE with a short distance between DUE pieces, while fullduplex (PSDC) is even more energy-efficient than full-duplex (PS). Simulation results show that, under the advanced SI cancellation techniques of PSDC, the full-duplex D2D mode can achieve $36 \%$ enhancement on EE compared to the halfduplex D2D mode with a short distance of D2D pair.

\section{Appendix}

\section{A. The Proof of Quasiconcavity}

Proof of Theorem 1. According to the function $f(a, p)=$ $\log _{2}(1+a p) /\left(\xi p+p_{c}\right)$ we defined, the first derivative of $f(a, p)$ with respect to $p$ is represented by

$$
\begin{aligned}
& \frac{d f(a, p)}{d p} \\
& =\frac{1}{P_{\text {tot }}^{2}}\left[a P_{\text {tot }} \frac{1}{\ln 2}\left(\frac{1}{1+a p}\right)-\xi \log _{2}(1+a p)\right],
\end{aligned}
$$

where

$$
\begin{aligned}
f_{1}(p) & =\frac{a P_{\text {tot }}}{\ln 2 \times(1+a p)}, \\
f_{2}(p) & =\xi \log _{2}(1+a p), \\
P_{\text {tot }} & =\xi p+p_{c} .
\end{aligned}
$$

The first derivative of $f_{1}(p)$ and $f_{2}(p)$ with respect to $p$ is represented by

$$
\begin{aligned}
& \frac{d f_{1}(p)}{d p}=\frac{a\left(\xi-a p_{c}\right)}{\ln 2 \times(1+a p)^{2}}, \\
& \frac{d f_{2}(p)}{d p}=\frac{a \xi}{\ln 2 \times(1+a p)} .
\end{aligned}
$$

Then, for $p \in[0,+\infty)$, we have

$$
\frac{d f_{1}(p)}{d p}-\frac{d f_{2}(p)}{d p}=-\frac{a^{2} p_{c}}{\ln 2 \times(1+a p)^{2}}<0 .
$$

Let $f_{3}(p)=f_{1}(p)-f_{2}(p)$. Then, we have $f_{3}(+\infty)<f_{3}(p)<$ $f_{3}(0), \forall p \in[0,+\infty)$. Since $\lim _{p \rightarrow+\infty} f_{3}(p)=-\infty<0$ and $\lim _{p \rightarrow 0} f_{3}(p)=a p_{c} / \ln 2>0$, there exists a single value of $p$ denoted as $p^{*}$, so that $f_{3}\left(p^{*}\right)=0$. Obviously, since the denominator $P_{\text {tot }}^{2}$ is positive, we have $d f(a, p) / d p>0$ when $p<p^{*}$ and $d f(a, p) / d p<0$ when $p>p^{*}$. It means that $f(a, p)$ first increases and then decreases when $p$ increases. In addition, it is easily verified that $d \log _{2}(1+a p) / d p=$ $a /(\ln 2(1+a p))>0$; thus, numerator $\log _{2}(1+a p)$ increases monotonically with $p$. Hence, numerator $\log _{2}(1+a p)$ is a concave function of $p$. Besides, the denominator $P_{\text {tot }}$ is an affine function of $p$. Therefore, $f(a, p)$ is strictly quasiconcave in $p$ for $p \in[0,+\infty)$. The proof of Theorem 1 is complete.

\section{B. The Proof of Existence of Nash Equilibrium}

Proof of Theorem 2. According to [36], a Nash equilibrium exists in a noncooperative game if the utility function is continuous and quasiconcave, and the set of strategies is a nonempty compact convex subset in the Euclidean space. Taking one of the DUE's EE objection functions, $\eta_{d 1 \text {-FD }}$ as an example, it has been proved that $\eta_{d 1 \text {-FD }}$ is quasiconcave in Appendix A. Obviously, the set of the power strategies is a nonempty compact convex subset in the Euclidean space $\mathfrak{R}^{L}$. It is also easily proved that the above conditions also hold for the cellular UE similarly. Therefore, a Nash equilibrium exists in the noncooperative game.

\section{Competing Interests}

The authors declare that they have no competing interests.

\section{Acknowledgments}

This work was supported in part by the National Natural Science Foundation of China (61172055, 61471135), the Guangxi Natural Science Foundation (2013GXNSFGA019004, 2015GXNSFBB139007), the Fund of Key Laboratory Cognitive Radio and Information Processing, Guilin University of Electronic Technology, China, and Guangxi Key Laboratory of Wireless Wideband Communication and Signal Processing (CRKL150104, CRKL160105), the Innovation Project of Guangxi Graduate Education (YCSZ2015144), and the Innovation Project of GUET Graduate Education (2016YJCX91).

\section{References}

[1] E. Hossain and M. Hasan, "5G cellular: key enabling technologies and research challenges," IEEE Instrumentation \& Measurement Magazine, vol. 18, no. 3, pp. 11-21, 2015.

[2] P. Demestichas, A. Georgakopoulos, D. Karvounas et al., "5G on the Horizon: key challenges for the radio-access network," IEEE Vehicular Technology Magazine, vol. 8, no. 3, pp. 47-53, 2013.

[3] A. Zakrzewska, S. Ruepp, and M. S. Berger, “Towards converged 5 G mobile networks-challenges and current trends," in Proceedings of the in ITU Kaleidoscope Academic Conference, pp. 39-45, Saint Petersburg, Russia, June 2014.

[4] S. Talwar, D. Choudhury, K. Dimou, E. Aryafar, B. Bangerter, and K. Stewart, "Enabling technologies and architectures for 5G wireless," in Proceedings of the IEEE MTT-S International Microwave Symposium (IMS '14), pp. 1-4, Tampa, Fla, USA, June 2014.

[5] G. Liu, F. R. Yu, H. Ji, V. C. M. Leung, and X. Li, "In-band fullduplex relaying: a survey, research issues and challenges," IEEE Communications Surveys and Tutorials, vol. 17, no. 2, pp. 500$524,2015$.

[6] Z. Zhang, K. Long, A. V. Vasilakos, and L. Hanzo, "Full-duplex wireless communications: challenges, solutions, and future research directions," Proceedings of the IEEE, vol. 104, no. 7, pp. 1369-1409, 2016.

[7] L. Song, Y. Li, and Z. Han, "Resource allocation in full-duplex communications for future wireless networks," IEEE Wireless Communications, vol. 22, no. 4, pp. 88-96, 2015. 
[8] N. Reider and G. Fodor, "A distributed power control and mode selection algorithm for D2D communications," EURASIP Journal on Wireless Communications and Networking, vol. 2012, article 266, 2012.

[9] R. Bolla, R. Bruschi, F. Davoli, and F. Cucchietti, "Energy efficiency in the future internet: a survey of existing approaches and trends in energy-aware fixed network infrastructures," IEEE Communications Surveys and Tutorials, vol. 13, no. 2, pp. 223244, 2011.

[10] Z. Hasan, H. Boostanimehr, and V. K. Bhargava, "Green cellular networks: a survey, some research issues and challenges," IEEE Communications Surveys and Tutorials, vol. 13, no. 4, pp. 524540, 2011.

[11] L. Wang, F. Tian, T. Svensson, D. Feng, M. Song, and S. Li, "Exploiting full duplex for device-to-device communications in heterogeneous networks," IEEE Communications Magazine, vol. 53, no. 5, pp. 146-152, 2015.

[12] E. Ahmed, A. M. Eltawil, Z. Li, and B. A. Cetiner, "Full-duplex systems using multireconfigurable antennas," IEEE Transactions on Wireless Communications, vol. 14, no. 11, pp. 5971-5983, 2015.

[13] G. Liu, F. R. Yu, H. Ji, V. C. M. Leung, and X. Li, "In-band fullduplex relaying: a survey, research issues and challenges," IEEE Communications Surveys \& Tutorials, vol. 17, no. 2, pp. 500-524, 2015.

[14] Z. Wei, X. Zhu, S. Sun, Y. Huang, L. Dong, and Y. Jiang, "Fullduplex versus half-duplex amplify-and-forward relaying: which is more energy efficient in $60-\mathrm{GHz}$ dual-hop indoor wireless systems?" IEEE Journal on Selected Areas in Communications, vol. 33, no. 12, pp. 2936-2947, 2015.

[15] E. Everett, A. Sahai, and A. Sabharwal, "Passive self-interference suppression for full-duplex infrastructure nodes," IEEE Transactions on Wireless Communications, vol. 13, no. 2, pp. 680-694, 2014.

[16] M. Duarte, C. Dick, and A. Sabharwal, "Experiment-driven characterization of full-duplex wireless systems," IEEE Transactions on Wireless Communications, vol. 11, no. 12, pp. 4296-4307, 2012.

[17] D. Bharadia, E. McMilin, and S. Katti, "Full duplex radios," in Proceedings of the Annual Conference of the ACM Special Interest Group on Data Communication on the Applications, Technologies, Architectures, and Protocols for Computer Communication (ACM SIGCOMM '13), vol. 43, no. 4, pp. 375-386, Hong Kong, August 2013.

[18] S. Li and R. D. Murch, "An investigation into baseband techniques for single-channel full-duplex wireless communication systems," IEEE Transactions on Wireless Communications, vol. 13, no. 9, pp. 4794-4806, 2014.

[19] E. Ahmed and A. M. Eltawil, "All-Digital self-interference cancellation technique for full-duplex systems," IEEE Transactions on Wireless Communications, vol. 14, no. 7, pp. 3519-3532, 2015.

[20] K. T. Hemachandra, N. Rajatheva, and M. Latva-Aho, "Sum-rate analysis for full-duplex underlay device-to-device networks," in Proceedings of the IEEE Wireless Communications and Networking Conference (WCNC '14), pp. 514-519, April 2014.

[21] H. Bagheri, F. A. M. Bonomi, and M. Katz, "Spectral efficiency and throughput enhancement by full-duplex D2D communication in mobile clouds," in Proceedings of the European Wireless Conference, pp. 1-6, Budapest, Hungary, May 2015.

[22] S. Kim and W. Stark, "Full duplex device to device communication in cellular networks," in Proceedings of the 2014
International Conference on Computing, Networking and Communications (ICNC '14), pp. 721-725, San Diego, Calif, USA, February 2014.

[23] T. Yang, R. Zhang, X. Cheng, and L. Yang, "Resource sharing for device-to-device communications underlaying full-duplex cellular networks," in Proceedings of the IEEE International Conference on Communication Systems (ICCS '14), pp. 16-20, November 2014.

[24] W. Cheng, X. Zhang, and H. Zhang, "Optimal power allocation for full-duplex D2D communications over wireless cellular networks," in Proceedings of the IEEE Global Communications Conference (GLOBECOM '14), pp. 4764-4769, December 2014.

[25] G. Zhang, K. Yang, P. Liu, and J. Wei, "Power allocation for full-duplex relaying based D2D communication underlaying cellular networks," IEEE Transactions on Vehicular Technology, vol. 64, no. 10, pp. 4911-4916, 2015.

[26] C. Choi, S. Park, and D.-H. Cho, "User-cooperation scheme based on clustering for energy efficiency in cellular networks with D2D communication," in Proceedings of the 2014 25th IEEE Annual International Symposium on Personal, Indoor, and Mobile Radio Communication (IEEE PIMRC '14), pp. 1365-1369, Washington, DC, USA, September 2014.

[27] C. Xiong, G. Y. Li, S. Zhang, Y. Chen, and S. Xu, "Energy- and spectral-efficiency tradeoff in downlink OFDMA networks," IEEE Transactions on Wireless Communications, vol. 10, no. 11, pp. 3874-3886, 2011.

[28] L. Wei, R. Q. Hu, Y. Qian, and G. Wu, "Energy efficiency and spectrum efficiency of multihop device-to-device communications underlaying cellular networks," IEEE Transactions on Vehicular Technology, vol. 65, no. 1, pp. 367-380, 2016.

[29] Y. A. Sambo, M. Z. Shakir, K. A. Qaraqe, E. Serpedin, M. A. Imran, and B. Ahmed, "Energy efficiency improvements in HetNets by exploiting device-to-device communications," in Proceedings of the 22nd European Signal Processing Conference (EUSIPCO '14), pp. 151-155, Lisbon, Portugal, September 2014.

[30] F. Wang, C. Xu, L. Song, and Z. Han, "Energy-efficient resource allocation for device-to-device underlay communication," IEEE Transactions on Wireless Communications, vol. 14, no. 4, pp. 2082-2092, 2015.

[31] Z. Zhou, M. Dong, K. Ota, J. Wu, and T. Sato, "Energy efficiency and spectral efficiency tradeoff in device-to-device (D2D) communications," IEEE Wireless Communications Letters, vol. 3, no. 5, pp. 485-488, 2014.

[32] A. Zappone, B. Matthiesen, and E. A. Jorswieck, "Energyefficient MIMO overlay communications for device-to-device and cognitive radio systems," in Proceedings of the IEEE Wireless Communications and Networking Conference (WCNC '16), pp. 1-6, Doha, Qatar, April 2016.

[33] A. C. Cirik, Y. Rong, and Y. Hua, "Achievable rates of full-duplex MIMO radios in fast fading channels with imperfect channel estimation," IEEE Transactions on Signal Processing, vol. 62, no. 15, pp. 3874-3886, 2014.

[34] L. Song, Y. Liao, K. Bian, L. Song, and Z. Han, "Cross-layer protocol design for CSMA/CD in Full-duplex WiFi networks," IEEE Communications Letters, vol. 20, no. 4, pp. 792-795, 2016.

[35] A. C. Cirik, J. Zhang, M. Haardt, and Y. Hua, "Sum-rate maximization for bi-directional full-duplex MIMO systems under multiple linear constraints," in Proceedings of the 15th IEEE International Workshop on Signal Processing Advances in Wireless Communications (SPAWC '14), pp. 389-393, Toronto, Canada, June 2014. 
[36] M. Jain, J. I. Choi, T. Kim et al., "Practical, real-time, full duplex wireless," in Proceedings of the 17th Annual International Conference on Mobile Computing and Networking (MobiCom '11), pp. 301-312, September 2011.

[37] G. Miao, N. Himayat, G. Y. Li, and S. Talwar, "Distributed interference-aware energy-efficient power optimization," IEEE Transactions on Wireless Communications, vol. 10, no. 4, pp. 1323-1333, 2011.

[38] D. Gesbert, S. G. Kiani, A. Gjendemsj, and G. E. Ien, "Adaptation, coordination, and distributed resource allocation in interference-limited wireless networks," Proceedings of the IEEE, vol. 95, no. 12, pp. 2393-2409, 2007.

[39] Z. Juntao, F. Wei, Z. Ming, and W. Jing, "Coordinated multi-user spectrum sharing in distributed antenna-based cognitive radio systems," China Communications, vol. 13, no. 1, pp. 57-67, 2016.

[40] Y. Zhao, Y. Li, H. Zhang, N. Ge, and J. Lu, "Fundamental tradeoffs on energy-aware D2D communication underlaying cellular networks: a dynamic graph approach," IEEE Journal on Selected Areas in Communications, vol. 34, no. 4, pp. 864-882, 2016.

[41] M. Sheng, Y. Li, X. Wang, J. Li, and Y. Shi, "Energy efficiency and delay tradeoff in device-to-device communications underlaying cellular networks," IEEE Journal on Selected Areas in Communications, vol. 34, no. 1, pp. 92-106, 2016. 

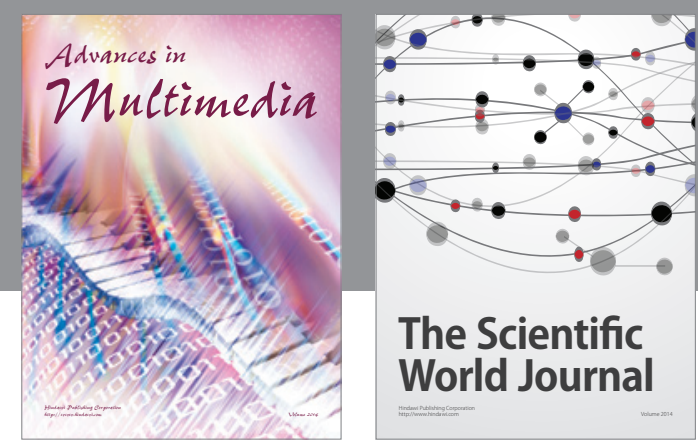

The Scientific World Journal
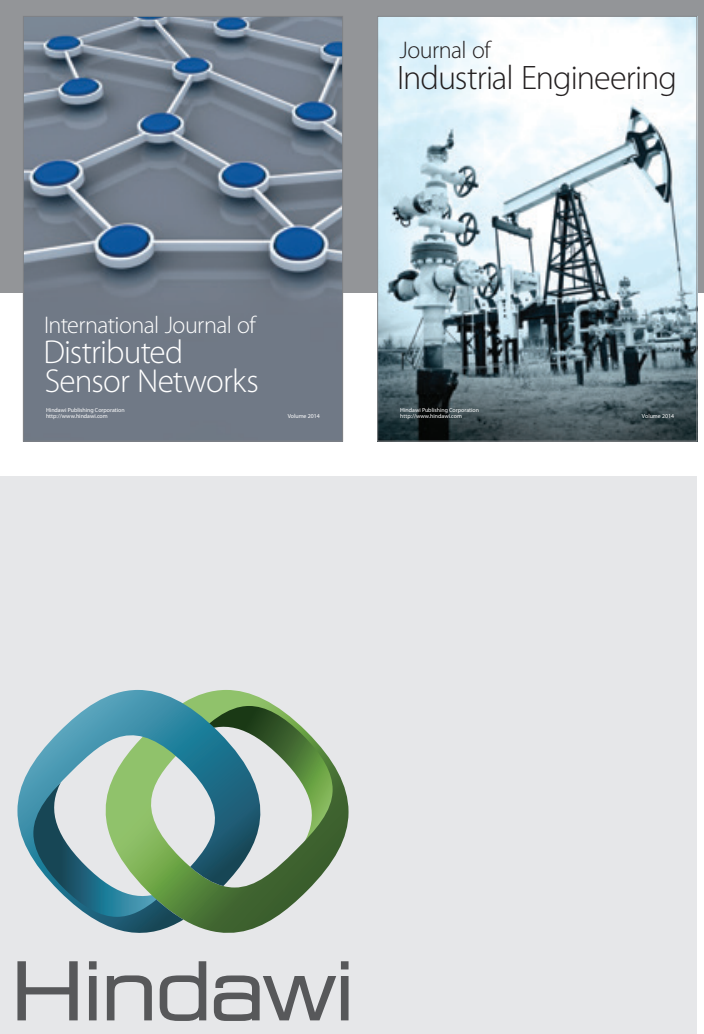

Submit your manuscripts at

http://www.hindawi.com

\section{Computer Networks} and Communications
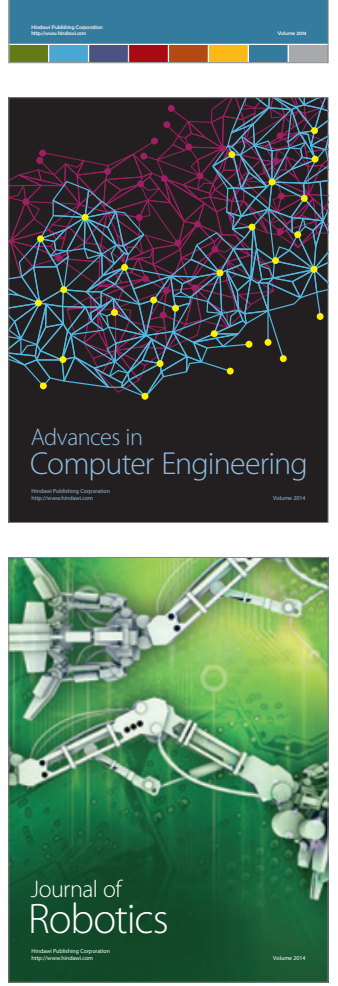
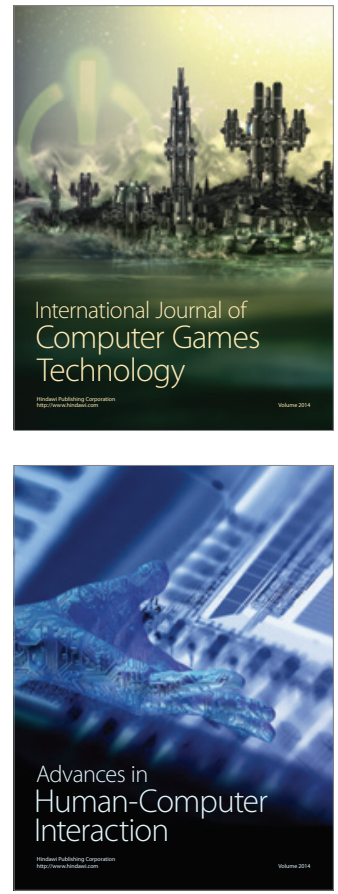
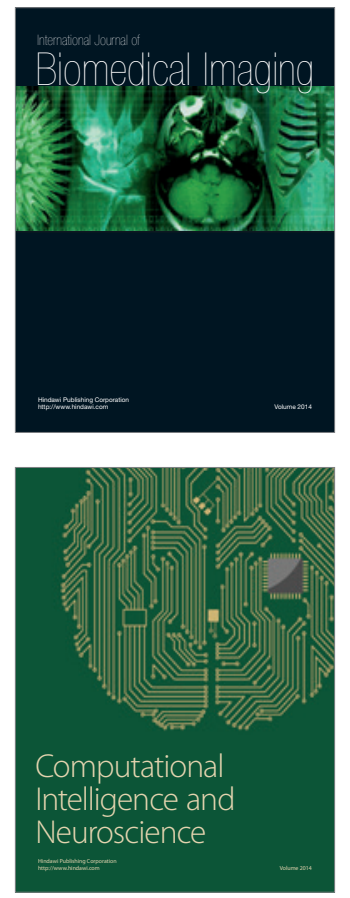
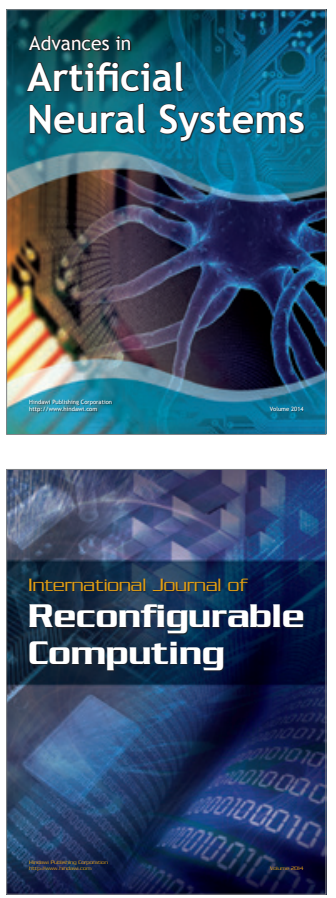
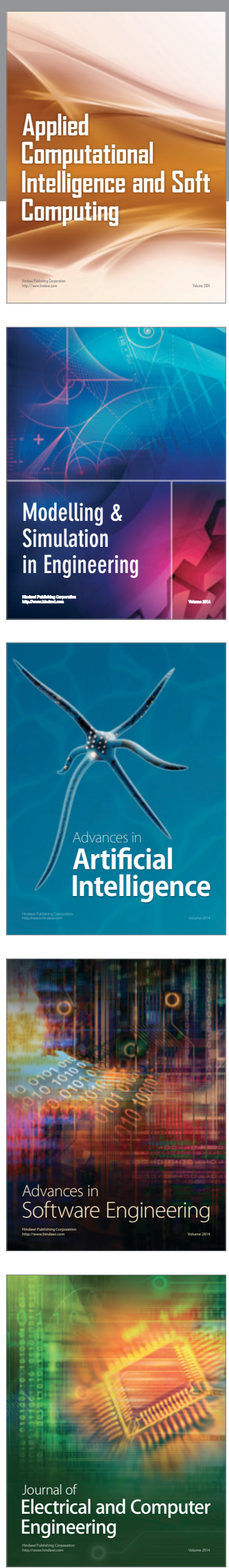